\title{
Electromagnetic Aspects of Neutral Elementary Particles in Relativistic Quantum Mechanics
}

\section{Jefferson Morais}

CBPF - Centro Brasileiro de Pesquisas Físicas

E-mail: moraisecbpf.br

\section{J. A. Helayël-Neto}

CBPF - Centro Brasileiro de Pesquisas Físicas

E-mail: helayel@cbpf.br

\section{Rodrigo Turcati*}

CBPF - Centro Brasileiro de Pesquisas Físicas

E-mail: turcatiecbpf.br

\begin{abstract}
This paper presents viable scenarios in which we can check the possibility that neutral genuinely elementary particles acquire a magnetic dipole moment at quantum-mechanical level and rather than as a loop effect in Quantum Field Theory. We actually have in mind to evaluate the very role of the spin for the magnetic properties of the particles, trying to disconnect spin from electric charge. For that, we adopt the Dirac equation for the description of massive charged, neutral or, eventually, self-conjugated (Majorana-like) fermions. We propose a sistematic study of nonminimal electromagnetic couplings and their consequences, in the non-relativistic regime, on the magnetic dipole moment generation for fermionic particles. The discussion sets out also in situations where Lorentz-symmetry breaking takes place. The investigation is also pursued for massive neutral vector bosons and we contemplate situations in which a non-trivial magnetic moment appears already at the tree-approximation, despite the absence of an electric charge for the particles under consideration.
\end{abstract}

5th International School on Field Theory and Gravitation, April 20 - 242009

Cuiabá city, Brazil

*Poster section 


\section{Introduction}

Modern physics is based on two pillars: The Standard Model of Particle Physics (SM) and General Relativity. The SM describes with great precision all fundamental particles and their strong, weak and electromagnetic interactions. On the other hand, General Relativity associates a geometry to gravitation, that is an excellent description at the classical level. But the attempt of unification of fundamental interactions, serious problems arise, such as renormalization. To date, no physical theory was able to show fully consistent with a scenario unifying at the fundamental level. Within this idea, Kostelecky and collaborators [1] proposed the possibility that symmetries considered fundamental, such as Lorentz-symmetry, were violated. A violation of Lorentz-symmetry would be held by background Lorentz tensor and vector fields, these account for an anisotropy space-time. The mechanism used for this violation is the fact the Lorentz tensor objects would take non-vanishing vacuum expected values, and thus induce spontaneous symmetry breaking. This possibility was obtained by Kostelecky in a context set by String Theory. After, other mechanisms for Lorentz-violation at fundamental level have been found, including Loop Quantum Gravity, Massive Gravity, Noncommutative field theories, among others. This environment is known as the Standard Model Extension (SME). It is a gauge invariant effective field theory that maintains the $S U(3) X S U(2) X U(1)$-structure. Within this context, the main goal of this work is the study of electromagnetic properties that neutral elementary particles might acquire when coupled to external electromagnetic fields responsible for the violation of Lorentz-symmetry. The aim is then to investigate the role of spin in the magnetic properties particles, decoupling it from the electric charge. Performing non-minimal interactions with relativistic particles of spin- $\frac{1}{2}$ and spin- 1 , we obtain the non-relativistic limit, and observe whether there is the appearance of electromagnetic properties, as topological quantum effects and generating of magnetic dipole moment. The procedure is based on Relativistic Quantum Mechanics [2].

\section{Massive Fermions coupled to Tensorial Fields and Non-Relativistic Interactions}

The starting point is the gauge invariant lagrangean density not-minimally coupled to tensor and vector fields, according to the expression below $\left(\eta_{\mu v}=\operatorname{diag}(1,-1,-1,-1)\right)(\hbar=c=1)$ :

$$
\begin{aligned}
\mathscr{L}= & \bar{\Psi}\left(\imath \gamma^{\mu} \partial_{\mu}-m\right) \Psi-e \bar{\Psi} \gamma^{\mu} \Psi A_{\mu}+f \bar{\Psi} \gamma^{\mu} \gamma_{5} \Psi H_{\mu}+i g \bar{\Psi} \Sigma^{\mu v} \Psi S_{\mu v}+ \\
& +h \bar{\Psi} \Sigma^{\mu v} \gamma_{5} \Psi G_{\mu v}+\alpha \bar{\Psi} \Psi \varphi+\beta \bar{\Psi} \gamma_{5} \Psi s,
\end{aligned}
$$

The tensor and vector fields in the lagrangean density are responsible for the Lorentz-symmetry violation of the charged spin- $\frac{1}{2}$ particle. We are interested in electromagnetic properties that elementary particles may acquire whenever coupled to the anisotropic background. To do that, we implement the Pauli coupling, i.e., substitute the tensor and pseudo-tensor fields by electromagnetic field $\left(S_{\mu \nu}=G_{\mu \nu}=F_{\mu \nu}\right)$. The coupling to the vector field, $A^{\mu}$, represents the minimal coupling with the electromagnetism and is responsible for the electric charge. This term does not violate Lorentzsymmetry, while the pseudo-vector $H^{\mu}$ does. The scalar and pseudo-scalar couplings represent the Higgs coupling in the fermion sector. As previously stated, our approach is based in the nonrelativistic limit. Starting from lagrangean density, we apply the variational method to obtain the relativistic field equation. After, we choose the Dirac representation and use the low-energy limit, 
i.e., the limit such that $E \simeq m(\mid \vec{p} \ll E)$ and obtain the Pauli-type equation. To begin our study, we adopt a more pedagogical view. To do that, we first consider the Dirac particle in the presence of a external eletrostatic field $\left(F_{0 i}=\vec{E}_{i}\right)$. Taking the non-relativistic limit, we obtain the Pauli-type equation to the electron:

$$
\imath \frac{\partial \psi}{\partial t}=\left\{\frac{\vec{\Pi}^{2}}{2 m}-\vec{\mu} \cdot \vec{B}+e \phi+H_{n m}\right\} \psi,
$$

where the generalized canonical momentum is given by:

$$
\vec{\Pi}=\left(-l \vec{\nabla}-e \vec{A}+\frac{4 g m}{e} \vec{\mu} \times \vec{E}-\frac{2 m f}{e} H^{0} \vec{\mu}\right) .
$$

and the non-minimal interactions are:

$$
H_{n m}=-\alpha \varphi+\frac{2 m}{e} \vec{\mu} \cdot(f \vec{H}-2 h \vec{E}) .
$$

We can see from the canonical momentum, besides the usual Aharonov-Bohm $(\mathrm{AB}) \operatorname{effect}(e \vec{A})[[]$ originating from the minimal electromagnetic coupling with the vector potential $A^{\mu}$, an AharonovCasher-type (AC) 㺻 phase $\left(\frac{4 g m}{e} \vec{\mu} \times \vec{E}\right)$ is induced by an external eletrostatic field. Here, the interesting fact is that the topological quantum effect is generated by a background field responsible for the Lorentz-symmetry violation.

Now considering the presence of a external magnetostatic field $\left(F_{i j}=-\varepsilon_{i j k} \vec{B}_{k}\right)$, we obtain in the low-energy limit:

$$
\begin{aligned}
l \frac{\partial \psi}{\partial t}= & \frac{1}{2 m}\left(-\imath \vec{\nabla}-e \vec{A}+\frac{4 h m}{e} \vec{\mu} \times \vec{B}-\frac{2 m f}{e} H^{0} \vec{\mu}\right)^{2} \psi-\vec{\mu} \cdot \vec{B} \psi+e \phi \psi+ \\
& -\alpha \varphi \psi+\frac{2 m}{e} \vec{\mu} \cdot(2 g \vec{B} \psi+f \vec{H}) \psi
\end{aligned}
$$

Here, we can see a correction to the magnetic dipole moment of the electron induced by an alignement of the fermion spin with the external magnetic field $\vec{B}$. It is curious that this correction appears already in Relativistic Quantum Mechanics, and not in Quantum Field Theory, by means of quantum loops corrections.

\section{Topological Quantum Effects and the Generation of Magnetic Moment for Neutral Elementary Particles}

Neutrino magnetic properties have been a subject of renewed interest over the past years. The Standard Model Extension is a good framework to set up this kind of conjecture. To do that, we shall treat the case of neutral elementary particles of spin- $\frac{1}{2}$, more specifically, the Majorana fermions coupled to tensor and vector fields responsible for the Lorentz-violation. In so doing, the lagrangean density assumes the form:

$$
\mathscr{L}=\bar{\Psi}\left({ }_{l} \gamma^{\mu} \partial_{\mu}-m\right) \Psi+f \bar{\Psi} \gamma^{\mu} \gamma_{5} \Psi H_{\mu}+h \bar{\Psi} \Sigma^{\mu v} \gamma_{5} \Psi F_{\mu v}+\alpha \bar{\Psi} \Psi \varphi+\beta \bar{\Psi} \gamma_{5} \Psi s
$$


As we did in the previous section, here we will study first the case of a external electrostatic field acting on a Majorana fermion. Thus, the Pauli-type equation is written as:

$$
\imath \frac{\partial \psi}{\partial t}=\left\{\frac{1}{2 m}\left(-\imath \vec{\nabla}+2 h \vec{\sigma} \times \vec{B}-f H^{0} \vec{\sigma}\right)^{2}-\alpha \varphi+f \vec{\sigma} \cdot \vec{H}\right\} \psi .
$$

Contrary to the Dirac fermion, an Aharonov-Casher phase does not show up now. However, there exist topological quantum effects originating from the torque between the spin and the magnetic field. But, we still are interested in the magnetic dipole moment to the neutral elementary particle of spin- $\frac{1}{2}$ can acquire. So, we place the particle in the presence of a external magnetostatic field. So, we obtain:

$$
\imath \frac{\partial \psi}{\partial t}=\left\{\frac{1}{2 m}\left(-\imath \vec{\nabla}-f H^{0} \vec{\sigma}\right)^{2}-\alpha \varphi+\vec{\sigma} \cdot(f \vec{H}-2 h \vec{E})\right\} \psi
$$

thought of as notice first that the term $\Sigma_{\mu \nu} \gamma_{5} F^{\mu v}$ in the Dirac representation is equivalent to $2 i \vec{\sigma} \cdot \vec{B}$. It could be thought as the interaction of the spin of Majorana fermion with the external magnetostatic field, featuring a magnetic dipole moment. But, the operator couples to both the dominant and weak components of relativistic fermion. In this way, this interpretation is not valid, and we need to analise the low-energy limit. Analising the above equation, we see no magnetic moment is induced. When we perform the study of non-minimal interactions that violate Lorentz-symmetry in the Relativistic Quantum Mechanics, we see that neutral elementary particles of spin- $\frac{1}{2}$ don't acquire any interesting electromagnetic property, such as a AC phase or a magnetic moment.

\section{Gauge Models with Lorentz-Symmetry Violation}

In this section, we are interested in the implementation of the Chern-Simons-type igv ${ }^{\alpha} \tilde{F}_{\mu \alpha}$ coupling in the massive spin-1 field non eletrical charged $(e=0)$. This term couples the dual tensor of electromagnetism to a background vector $v^{\mu}$, maintaining gauge invariance, but violating Lorentz-symmetry and Parity [5]. Being gauge invariant, we can introduce the non-minimal coupling directly in the covariant derivate operator according the expression below:

$$
\left(\partial_{\mu}+i g v^{\alpha} \widetilde{F}_{\mu \alpha}\right) Z^{\mu v}+m^{2} Z^{v}=0,
$$

where the $Z^{\mu}$ is the massive neutral vectorial boson and $Z^{\mu v}$ is the field strenght. As before, we are interested in the non-relativistic limit. To do this, we obtain the subsidiary condition:

$$
\partial_{\mu} Z^{\mu}+i g v^{\alpha} \widetilde{F}_{\mu \alpha} Z^{\mu}=0 .
$$

Now, putting the vectorial field $Z^{\mu}$ in the presence of a external eletrostatic field and expressing the subsidiary condition in its components in the non-relativistic limit, we get that:

$$
\begin{aligned}
Z^{0} & \approx \frac{\vec{p}}{m} \cdot \vec{Z}-\frac{g}{m}(\vec{v} \times \vec{E}) \cdot \vec{Z} \\
E_{N R} Z_{i} & =\frac{1}{2 m}\left[\vec{p}+\frac{g}{2}(\vec{v} \times \vec{E})\right]^{2} Z_{i} .
\end{aligned}
$$


In this limit, we observe that the $Z^{0}$ component tends to zero because the term $\frac{1}{m}$ is very small in this range. However, the spatial component $Z^{i}$ survives, showing that in the low-energy limit only the spatial part of massive spin-1 field is propagated. Here, we can observe how the non-trivial Aharonov-Casher phase $\left(\frac{g}{2}(\vec{v} \times \vec{E})\right)$ looks like. This result suggests that the quantity $\frac{1}{2} g \vec{v}$ can be interpreted as the magnetic moment acquired by the neutral particle due to the presence of the spatial component of the background vector $v^{\mu}$. To see if it corresponds to a magnetic moment, we switch off the electric field and switch on the magnetic field. The components of field equations are expressed as:

$$
\begin{aligned}
Z^{0} & \approx \frac{\vec{p}}{m} \cdot \vec{Z}-\frac{g v^{o}}{m} \vec{B} \cdot \vec{Z} \\
E_{N R} Z_{i} & =\frac{\vec{p}^{2}}{2 m} Z_{i}+\frac{1}{2} g \vec{v} \cdot \vec{B} Z_{i} .
\end{aligned}
$$

Again, only the spatial part propagates. We see that the quantity $\frac{1}{2} g \vec{\mu}$ really corresponds a magnetic dipole moment. Previous works [6] have shown that this magnetic moment induced by a ChernSimons term can be thought as a universal magnetic moment acquired by the neutral elementary particles independent of their spin, i.e., all the elementary particles that interact with the background vector, $v^{\mu}$, must be endowed with a magnetic moment that is independent of its electric charge. This result is similar to the electric charge acquired by the particles when coupled to the electromagnetic field in a minimal way.

Another possibility is relative to the $k_{F}$-parameter, well-known in the literature of the Standard Model Extension [7]. It has the property to be CPT-invariant, but it violates the Lorentz-symmetry. Whenever inserted in the dynamic equation of the quantity $Z^{\mu}$, we have:

$$
D_{\mu} Z^{\mu v}-\frac{1}{2} k_{F}^{v \alpha \lambda \rho} D_{\alpha} Z_{\lambda \rho}+m^{2} Z^{v}=0
$$

where the covariant derivate operator is $D_{\mu}=\partial_{\mu}+i g v^{\alpha} \tilde{F}_{\mu \alpha}$. Adopting the non-relativistic limit, we obtain that the contribution to the magnetic moment originated from $k_{F}$-parameter is:

$$
\vec{\mu}_{i j} \cdot \vec{B} Z_{j}
$$

where the nth component of the $\vec{\mu}_{i j}$ is given by

$$
\left(\mu_{n}\right)_{i j}=\frac{g}{2} v^{0}\left(k_{F}\right)_{n i j 0},
$$

The correction to magnetic moment induced by the $k_{F}$-parameter is relative to temporal component of the background vector $v^{\mu}$ instead the spatial component as in the previous case.

\section{Conclusion}

Despite the great success of the Standard Model of Particle Physics, the search for a theory that explains in a unified way the fundamental interactions, motivated studies related to the violation of CPT-theorem, leading to the creation of a field of knowledge known as the Standard Model Extension. Within this view, this study examined the contributions of magnetic dipole moment of 
neutral elementary particles where there was Lorentz-symmetry violation, from the perspective of Relativistic Quantum Theory, i.e., without quantization of fields. Small corrections for the magnetic moment of elementary particles could be an indication of Lorentz-symmetry violation and CPTinvariance. In the SM, the corrections for the magnetic moment come from quantum loop effects of Quantum Field Theory. In this context, it is necessary that the fields express their quantum character to be noticed any difference that the magnetic moment could present. In the context of the SME, which uses the aspect of Lorentz-symmetry violation, the effects of magnetic moment are already present in the Relativistic Quantum Mechanics. Although Lorentz-symmetry violation appears to manifest at very high energies, its effects can be observed in the low-energy limit. This suggests the possibility that these violations have a fundamental character as atributions of physical properties of elementary particles.

It is known that, in Nature, we find examples of systems that violate parity, charge conjugation and time reversal. However, in Quantum Field Theory, Lorentz-symmetry and CPT-invariance are valid under well-defined conditions, and no case of CPT-violation was observed to date. However, the violation of this theorem opens a path whose goal would be to construct a theory able to explain the interactions in a unified way. Therefore, many studies and problems are still open, offering the prospect of new discoveries in relation to the world of particles. Among these possibilities, it emphasizes the study of particles with different spins, as the graviton. Classical effects also can be investigated, as the existence of a Lorentz force. A series of questions are still seeking a satisfactory answer as the source of the Lorentz-symmetry and CPT-violation, a problem with a solution already routed via most fundamental theories, such as String Theory.

\section{Acknowledgements}

Jefferson Morais, J. A. Helayël-Neto and Rodrigo Turcati are grateful to CNPq for the financial help.

\section{References}

[1] V.A. Kostelecky and S. Samuel, Spontaneous breaking of Lorentz symmetry in string theory, Physical Review D 39(2):683-685, 1989

[2] J.D. Bjorken and S.D. Drell, Relativistic Quantum Mechanics, New York 1964

[3] Y. Aharonov and D. Bohm, Significance of Electromagnetic Potentials in the Quantum Theory, Physical Review 115(3):485-491, 1959

[4] Y. Aharonov and A. Casher, Topological Quantum Effects for Neutral Particles, Phys. Rev. Lett. 53:319, 1984

[5] S.M. Carroll, G.B. Field, and R. Jackiw, Limits on a Lorentz-and parity-violating modification of electrodynamics, Physical Review D 41(4):1231-1240, 1990

[6] H. Belich, LP Colatto, T. Costa-Soares, JA Helayël-Neto, and MTD Orlando, Magnetic Moment Generation from non-minimal couplings in a scenario with Lorentz-Symmetry Violation, Eur. Phys. J. C 62:425-432, 2009

[7] Quentin G. Bailey and V. Alan Kostelecky, Lorentz-violating electrostatics and magnetostatics, Physical Review D 70:076006, 2004 